Pacific Journal of Mathematics

ON AN ANALOGUE OF THE WIENER TAUBERIAN THEOREM
FOR SYMMETRIC SPACES OF THE NONCOMPACT TYPE 


\title{
ON AN ANALOGUE OF THE WIENER TAUBERIAN THEOREM FOR SYMMETRIC SPACES OF THE NON-COMPACT TYPE
}

\author{
Alladi Sitaram
}

\begin{abstract}
Let $X$ be a symmetric space and $G$ the connected component of the group of isometries of $X$. If $f \in L^{1}(X)$, we consider conditions under which $\operatorname{Sp}\left\{{ }^{g} f: g \in G\right\}$ is dense in $L^{1}(X)$ in terms of the "Fourier transform" of $f$. This continues earlier work on this kind of problem by L. Ehrenpreis and F. I. Mautner, R. Krier and the author.
\end{abstract}

1. Introduction. Let $f$ be an integrable function on $R$. Then we have the famous Wiener-Tauberian theorem: If the Fourier transform $\hat{f}$ is a nowhere vanishing function on $R$, then the ideal generated by $f$ is dense in $L^{1}(R)$. In [EM1] Ehrenpreis and Mautner observed that the exact analogue of the above theorem is no longer true if one considers the commutative Banach algebra of $K$-bi-invariant $L^{1}$-functions on a non-compact semi-simple Lie group $G$, where $K$ is a maximal compact subgroup of $G$-i.e. Let $I_{1}(G)$ denote the commutative Banach algebra of $K$-bi-invariant $L^{1}$-functions on $G$. For $f \in I_{1}(G)$ let $\hat{f}$ denote its spherical Fourier transform (see $\S 2$ ). Then there exist functions $f \in I_{1}(G)$ such that $\hat{f}$ is nowhere vanishing on the maximal ideal space $M$ of $I_{1}(G)$ and yet the algebra generated by $f$ is not dense in $I_{1}(G)$. However when $G=\operatorname{SL}(2, R)$ they were able to show that a modified version of Wiener's theorem is true i.e. $\hat{f}$ nowhere vanishing on $M$ together with the condition that "it does not go to zero too fast at $\infty$ " would indeed imply that the ideal generated by $f$ is dense in $I_{1}(G)$. (Theorems 6 and 7 of [EM1].) This kind of result has been generalized by R. Krier [K1] when $G$ is a non-compact connected semisimple Lie group of real rank 1 and by the author for $G$ of arbitrary rank [Si].

However the problem becomes considerably more difficult if one asks the following question: Let $f_{1}, f_{2}, \ldots, f_{n}$ be functions in $I_{1}(G)$ such that their spherical Fourier transforms have no common zeros in $M$. If $\left|\hat{f}_{1}\right|+\left|\hat{f}_{2}\right|+\cdots+\left|\hat{f}_{n}\right|$ "does not go to zero too fast at $\infty$ ", then is the ideal generated by $f_{1}, \ldots, f_{n}$ dense in $I_{1}(G)$ ? In this paper we try to give an answer to this question when the rank of $G$ is $1-$ The 
main tool used in a non-trivial extension of the Corona theorem due to T. Wolff ([Gar]).

At this point it may be appropriate to point out one of the reasons why the problem becomes much harder for $n$ functions. If $f_{1}, f_{2}, \ldots, f_{n}$ are functions in $L^{1}(R)$ such that the Fourier transforms $\hat{f}_{1}, \hat{f}_{2}, \ldots, \hat{f}_{n}$ have no common zeros, then one can consider the function $g=f_{1}^{*} *$ $f_{1}+f_{2}^{*} * f_{2}+\cdots+f_{n}^{*} * f_{n}$. Then it is easy to see that $\hat{g}$ has no zero in $R$ and hence we can apply Wiener's theorem to the single function $g$. However this trick is not available to us in the case of $I_{1}(G)$. This is because in this case the maximal ideal space $M$ is a certain "strip" in the complex plane (see $\S 2$ ) and so while $\hat{g}$ will not have any real zeros, it may still have complex zeros in $M$.

Finally we look at the following problem: Suppose $X(=G / K)$ is a Symmetric Space of the non-compact type. Let $f \in L^{1}(X)$. Can one get an analogue of Wiener's theorem in this set up? i.e. Can one give conditions on the "Fourier transform of $f$ " which will ensure that the $G$-translates of $f$ will span a dense subspace of $L^{1}(X)$ ? We take up this question in $\S 4$ and observe that in the special case $f$ is $K$ bi-invariant, we already have some answers to this question. Further we use the results of $\S 3$ to give an answer to this question in the case $G=\operatorname{SL}(2, R)$ and $f$ a general left $K$-finite function in $L^{1}(X)$.

2. Notation and preliminaries. Unless otherwise stated, $G$ will denote a connected, non-compact, semi-simple Lie group with finite centre, of real rank 1 and $K$ a fixed maximal compact subgroup of $G$. Fix an Iwasawa decomposition $G=\mathrm{KAN}$ and let $\mathbf{a}$ be the Lie algebra of $A$. Let $\mathbf{a}^{*}$ be the real dual of $\mathbf{a}$ and $\mathbf{a}_{c}^{*}$ its complexification. Let $\rho$ be the half-sum of the positive roots for the adjoint action of a on the Lie algebra $\mathbf{g}$ of $G$. Since $\operatorname{dim} \mathbf{a}^{*}=1$, every element in $\mathbf{a}_{c}^{*}$ is of the form $\lambda \rho$ with $\lambda \in \mathbf{C}$. Thus we can identify $\mathbf{a}_{c}^{*}$ with $\mathbf{C}$ (via this identification).

For each $\lambda \in \mathbf{a}_{c}^{*}(=\mathbf{C})$ let $\phi_{\lambda}$ be the elementary spherical function associated with $\lambda$ (see [H2] for details). Then it is known that $\phi_{\lambda}=\phi_{\lambda^{\prime}}$, iff $\lambda=\lambda^{\prime}$ or $\lambda=-\lambda^{\prime}$. Let $F=\left\{\lambda: \phi_{\lambda}\right.$ is a bounded function on $\left.G\right\}$. Then by a well known theorem of Helgason and Johnson [H2], $F=$ $R+i[-1,1]$ (where $i=\sqrt{-1}$ ).

Let $I(G)$ be the set of all complex valued spherical functions on $G$, i.e., $I(G)=\left\{f: f\left(k_{1} x k_{2}\right)=f(x), k_{1}, k_{2} \in K, x \in G\right\}$. Fix a Haar measure $d x$ on $G$ and let $I_{1}(G)=I(G) \cap L^{1}(G, d x)$. Then it is well known that $I_{1}(G)$ is a commutative Banach algebra under convolution and that the maximal ideal space of $I_{1}(G)$ can be identified with $F / W$ where $W$ is the two-element group acting on $F$ in the obvious 
way. We shall denote by $I^{\infty}(G)$ the space of $C^{\infty}$-spherical functions and by $I_{c}^{\infty}(G)$ the space of compactly supported functions in $I^{\infty}(G)$. Let $S_{1}(G)$ denote the $L^{1}$-Harish-Chandra-Schwartz space of spherical functions (i.e. the subspace of $I_{1}(G)$ which are rapidly decreasingfor a description of the topology on this space see [W]). Then $S_{1}(G)$ is a Fréchet space and $S_{1}(G) \rightarrow I_{1}(G)$ is a dense inclusion. Let $Z(F)$ be the space of even functions $f$ on $F$ satisfying:

(i) $f$ is holomorphic in $F^{0}$ (the interior of $F$ ) and continuous on $F$.

(ii) For all non-negative integers $l, k$,

$$
\sup _{\lambda \in F^{0}}\left[(1+|\lambda|)^{l}\left|\frac{d^{k} f}{d \lambda^{k}}(\lambda)\right|\right]<\infty .
$$

Then (ii) can be used in the obvious way to define a family of seminorms on $Z(F)$ under which $Z(F)$ becomes a Fréchet space. Now the well known theorem of Trombi-Varadarajan [TV] says that $h \rightarrow \hat{h}$ is a topological isomorphism from $S_{1}(G)$ onto $Z(F)$. Here, for any $h \in I_{1}(G)$, the spherical Fourier transform $\hat{h}$ is defined on $F$ by:

$$
\hat{h}(\lambda)=\int_{G} h(x) \phi_{-\lambda}(x) d x, \quad \lambda \in F .
$$

Then it is well known that $\hat{h}$ is an even, bounded, continuous function on $F$, holomorphic in $F^{0}$. Also $(f * g)^{\wedge}=\hat{f} \cdot \hat{g}$ for $f, g \in I_{1}(G)$ where $*$ denotes convolution in the group $G$ with respect to $d x$. If $f \in I_{c}^{\infty}(G)$ then $\hat{f}$ is well defined on all of $\mathbf{C}$ (and in fact will be an entire even function on $\mathbf{C}$ satisfying the Paley-Wiener growth condition).

For $\varepsilon>0$, let us denote by $F_{\varepsilon}$ the subset $R+i[-(1+\varepsilon),(1+\varepsilon)]$ of C. The following theorem has been proved by Ehrenpreis-Mautner for $\operatorname{SL}(2, R)$ in [EM1] and by R. Krier in [K1]. (For groups of arbitrary rank such a theorem has been proved by the author in [Si].):

Theorem A. Let $f \in I_{1}(G), \hat{f}(Z) \neq 0$ for $Z \in F, \hat{f}$ twice continuously differentiable in the closed band $F$ with bounded second derivative in $F$ and

$$
\sup _{Z \in F}\left|\frac{d^{k}}{d Z^{k}}\left((\hat{f}(Z))^{-1} e^{-Z^{2 p}}\right)\right|<\infty \quad \text { for } k=0,1,2 \text {, and some } p \in \mathbf{Z}^{+} \text {. }
$$

Then the ideal generated by $f$ is dense in $I_{1}(G)$.

Note. The parametrization adopted by Ehrenpreis-Mautner is different from ours-this accounts for the factor $e^{-s^{4 n}}$ rather than $e^{-Z^{2 n}}$ 
in their work. For them, in the variable $s=x+i y$, it is $y$ that goes to $\pm \infty$. So it is clear that they require, for rapid decay at $\infty$, the factor $-(x+i y)^{4 n}$ (rather than $\left.-(x+i y)^{2 n}\right)$.

The following non-trivial extension of the Corona theorem due to T. Wolff ([Gar]) plays a crucial role in this paper.

TheOREM B. Let $F_{\varepsilon}^{0}$ denote the interior of $F_{\varepsilon}$, i.e., $F_{\varepsilon}^{0}$ is the open band $R+i(-(1+\varepsilon),(1+\varepsilon))$ and let $H^{\infty}\left(F_{\varepsilon}^{0}\right)$ denote the set of bounded holomorphic functions on $F_{\varepsilon}^{0}$. Let $g, f_{1}, f_{2}, \ldots, f_{n}$ belong to $H^{\infty}\left(F_{\varepsilon}^{0}\right)$ such that $|g(Z)| \leq \sum_{k=1}^{n}\left|f_{k}(Z)\right|$ for all $Z \in F_{\varepsilon}^{0}$. Then there are $g_{1}, g_{2}, \ldots, g_{n}$ in $H^{\infty}\left(F_{\varepsilon}^{0}\right)$ such that $g^{3}=g_{1} f_{1}+g_{2} f_{2}+\cdots+g_{n} f_{n}$ (i.e., $g^{3}$ is in the ideal generated by $f_{1}, \ldots, f_{n}$ in $\left.H^{\infty}\right)$.

(Remark: This problem is usually stated for the open unit disc but notice that $F_{\varepsilon}^{0}$ is conformally equivalent to the open unit disc.)

For any unexplained notation and terminology in this section, please see [H1].

3. An application of Theorems $\mathbf{A}$ and $\mathbf{B}$. Using Theorems $\mathbf{A}$ and $\mathbf{B}$ of $\S 2$, we can now prove the result mentioned in the introduction. We continue to assume that rank of $G$ is 1 .

THEOREM 3.1. Let $f_{1}, f_{2}, \ldots, f_{n} \in I_{1}(G)$. Suppose for some $\varepsilon>$ $0, \hat{f}_{1}, \hat{f}_{2}, \ldots, \hat{f}_{n}$ extend to bounded holomorphic functions on $F_{\varepsilon}^{0}$ and there exists a positive constant $K$ and a positive integer $l$ such that $\sum_{j=1}^{n}\left|\hat{f}_{j}(Z)\right| \geq K\left|e^{-Z^{2 l}}\right|, Z \in F_{\varepsilon}^{0}$, then the ideal generated by $f_{1}, f_{2}, \ldots$, $f_{n}$ in $I_{1}(G)$ is dense in $I_{1}(G)$.

Proof. (Notice that in any case $\hat{f}_{1}, \hat{f}_{2}, \ldots, \hat{f}_{n}$ are in $H^{\infty}\left(F^{0}\right)$, continuous on $F$ and the condition $\sum_{j=1}^{n}\left|f_{j}(Z)\right| \geq K\left|e^{-Z^{2 l}}\right|$ in $F_{\varepsilon}^{0}$ implies that the $f_{j}$ 's have no common zeros in $F$.) By Wolff's theorem (Theorem B in §2) there exist bounded holomorphic functions $g_{1}, g_{2}, \ldots, g_{n}$ on $F_{\varepsilon}^{0}$ such that

$$
g_{1} \hat{f}_{1}+g_{2} \hat{f}_{2}+\cdots+g_{n} \hat{f}_{n}=\left(K e^{-Z^{2 l}}\right)^{3}=K^{3} e^{-3 Z^{2 l}} .
$$

Since the $\hat{f}_{j}$ and $e^{-Z^{2 l}}$ are even functions on $F_{\varepsilon}^{0}$, we can assume that the $g_{j}$ are also even (otherwise replace $g_{j}(Z)$ by $\left(g_{j}(Z)+g_{j}(-Z)\right) / 2$ ). Thus: $e^{-Z^{2}} g_{1} \hat{f}_{1}+\cdots+e^{-Z^{2}} g_{n} \hat{f}_{n}=K^{3} e^{-3 Z^{2 l}} e^{-Z^{2}}$. Since the $g_{j}$ are bounded holomorphic functions on $F_{\varepsilon}^{0}$ it is easy to see that $e^{-Z^{2}} g_{j}$ are rapidly decreasing in the smaller strip $F$ i.e. is in $Z(F)$ (see $\S 2$ for definition). Thus it follows from the Trombi-Varadarajan theorem 
that $\exists h_{j} \in S_{1}(G)$ such that $\hat{h}_{j}=e^{-Z^{2}} g_{j}$. Also for the same reason $K^{3} e^{-3 Z^{2 l}} e^{-Z^{2}}=\hat{h}$ for some $h \in S_{1}(G)$. Also note that $h$ satisfies the condition of Theorem A (take $p>l$ ). Hence the ideal generated by $h$ is dense in $I_{1}(G)$. Thus we have:

$$
\hat{h}_{1} \hat{f}_{1}+\cdots+\hat{h}_{n} \hat{f}_{n}=\hat{h}
$$

i.e.

$$
h_{1} * f_{1}+\cdots+h_{n} * f_{n}=h
$$

and since $h$ generates an ideal dense in $I_{1}(G)$, from the above equation it follows that the ideal generated by $f_{1}, \ldots, f_{n}$ is dense in $I_{1}(G)$. (As M. Cowling pointed out to us, it is possible to use the Corona theorem directly but the proof becomes simpler if one uses Wolff's extension of the Corona theorem.)

4. Symmetric spaces. Let $G$ and $K$ be as before, except that for the moment we do not make any assumptions on the rank of $G$. Let $X=G / K$ be the corresponding Symmetric Space of the non-compact type and denote by $L^{1}(X)=L^{1}(G / K)$ the subspace of $L^{1}(G)$ of right $K$-invariant functions. Now $G$ acts on $X$ by left action and hence on $L^{1}(X)$ by left regular action. Notice we have $I_{1}(G) \subset L^{1}(X) \subset L^{1}(G)$, a chain of closed subspaces. The problem considered in the previous section can be viewed now in a slightly wider context. Given $f \in I_{1}(G)$, we have shown that under certain conditions on the spherical Fourier transform $\hat{f}_{1}$ the ideal generated by $f$ is dense in $I_{1}(G)$ this is equivalent to saying that the left $G$-translates of $f$ span a dense subspace of $L^{1}(X)$. (This follows from the well known equivalence between closed left $G$-invariant subspaces and closed subspaces invariant under left convolution and the fact that the smallest closed left $G$-invariant subspace of $L^{1}(G)$ containing $I_{1}(G)$ is $L^{1}(X)$.) So a natural question to ask is: Given $f \in L^{1}(X)$, give conditions on the "Fourier transform" of $f$ to ensure that the left $G$-translates of $f$ span a dense subspace of $L^{1}(X)$. Motivated by results for $I_{1}(G)$ we make a conjecture regarding this and observe that the conjecture holds in certain special cases. Before formulating the conjecture we need to introduce some more notation-for this we have found the exposition in the introduction of [BeS] very useful and we follow this very closely.

Consider as before the Iwasawa decomposition $G=\mathrm{KAN}$ and let $M$ be the centralizer of $A$ in $K$. For $\lambda \in \mathbf{a}_{c}^{*}$, let $\pi_{\lambda}$ be the spherical 
principal series representation of $G$ on $L^{2}(K / M)$ given by:

$$
\left(\pi_{\lambda}(x) V\right)(b)=e^{(i \lambda-\rho) H\left(x^{-1} b\right)} V\left(k\left(x^{-1} b\right)\right), \quad b \in K / M,
$$

where we use the Iwasawa decomposition

$$
y=k(y) \cdot \exp H(y) \cdot n(y) \in \mathrm{KAN}, \quad y \in G,
$$

and $\rho$ denotes as usual half of the sum of the positive restricted roots. Then one knows that for the Harmonic Analysis on $G / K$, these are the only relevant representations of $G$. Further for $\lambda \in \mathbf{a}^{*}, \pi_{\lambda}$ is unitary and irreducible. One can show that for $f \in L^{1}(X)$ and $\lambda \in \mathbf{a}^{*}$, the "Fourier transform":

$$
\lambda \rightarrow \pi_{\lambda}(f)=\int_{G} f(x) \pi_{\lambda}(x) d x
$$

is the bounded linear operator on $L^{2}(K / M)$ given by

$$
\left(\pi_{\lambda}(f) V\right)(b)=\left(\int_{K / M} V(u) d u\right) \int_{G} e^{(i \lambda-\rho) H\left(g^{-1} b\right)} f(g) d g .
$$

(Thus we can identify, if we choose, $\pi_{\lambda}(f)$ with the function $\tilde{f}$ on $\mathbf{a}^{*} \times$ $K / M$ given by: $\tilde{f}(\lambda, b)=\int_{G} e^{(i \lambda-\rho) H\left(g^{-1} b\right)} f(g) d g$-this is essentially Helgason's Fourier transform on a symmetric space, but we will not be using this identification.) Fix an orthonormal basis $V_{0}, V_{1}, V_{2}, \ldots$ of $L^{2}(K / M)$ such that each $V_{i}$ transforms according to some irreducible finite dimensional representation of $K$ and $V_{0}$ is the constant function 1 on $K / M$. Then one knows that for $f \in L^{1}(G / K), \pi_{\lambda}(f) V_{i}=0$ if $i \neq 0$ and thus $\pi_{\lambda}(f)$ is completely determined by its action on $V_{0}$. Now let us further assume that $f$ is left $K$-finite, (i.e. all the left translates of $f$ under $K$ lie in a finite dimensional subspace). Then if $f$ is a non-trivial function, for some non-trivial bounded continuous functions $a_{1}, a_{2}, \ldots, a_{n}$ and $i_{1}, i_{2}, \ldots, i_{n} \in \mathbf{Z}^{+} \cup\{0\}$, we have:

$$
\pi_{\lambda}(f) V_{0}=a_{1}(\lambda) V_{i_{1}}+\cdots+a_{n}(\lambda) V_{i_{n}} .
$$

Now

$$
a_{k}(\lambda)=\left\langle\pi_{\lambda}(f) V_{0}, V_{i_{k}}\right\rangle=\int_{G}\left\langle\pi_{\lambda}(x) V_{0}, V_{i_{k}}\right\rangle f(x) d x .
$$

Now one can show (by comparing with elementary spherical functions) that $x \rightarrow\left\langle\pi_{\lambda}(x) V_{0}, V_{i_{k}}\right\rangle$ is a bounded function on $G$ if $\lambda$ is in the "Helgason-Johnson" strip $F=\mathbf{a}^{*}+i C_{\rho}$, where $C_{\rho}=$ convex hull of $\{s \rho: s \in W\}$ and $W$ is the Weyl-group of the pair $(G, A)$. Thus from this one can show that, as for the spherical Fourier transform, each $a_{i}(\lambda)$ extends to a continuous function on $F$, holomorphic on 
$F^{0}$. (Note: We are not claiming that $\pi_{\lambda}$ is a uniformly bounded representation for $\lambda \in F$, but merely that each spherical function $x \rightarrow\left\langle\pi_{\lambda}(x) V_{0}, V_{i_{k}}\right\rangle$ is bounded-the question of which representations are uniformly bounded is much deeper. For recent work on this see [C], [St].) Thus if $f$ is left $K$-finite as above, we can extend the notion of $\pi_{\lambda}(f)$ for $\lambda$ not only in $\mathbf{a}^{*}$ but in $F$ by using $(*)$. Thus for each $\lambda \in F, \pi_{\lambda}(f)$ is a finite dimensional operator (of dimension $\leq n$ ) and further $\lambda \rightarrow \pi_{\lambda}(f)$ is holomorphic in $F^{0}$ and continuous on $F$. We are thus in a position to formulate our general conjecture:

(C): Retaining the above notation, if $\pi_{\lambda}(f)$ is nowhere vanishing (as an operator) for $\lambda \in F$ and if " $\left\|\pi_{\lambda}(f)\right\|$ does not go to zero too fast at $\infty$ ", then the left $G$-translates of $F$ span a dense subspace of $L^{1}(G / K)$.

REMARKS. (i) $\pi_{\lambda}(f)$ is nowhere vanishing as an operator for $\lambda \in F$ is equivalent to saying that the $n$-functions $a_{1}, \ldots, a_{n}$ do not have a common zero in $F$.

(ii) We are keeping the phrase "does not go to zero too fast at $\infty$ " imprecise for the moment.

(iii) If $V_{i_{k}} \neq V_{0}$, then $a_{k}(\lambda)$ necessarily vanishes at $-i \rho$. (This is because the function $\left\langle\pi_{-i \rho}(x) V_{0}, V_{i_{k}}\right\rangle$ is identically zero on $G$ if $V_{i_{k}} \neq$ $V_{0}$.) Therefore under the condition $\pi_{\lambda}(f)$ is nowhere vanishing on $\mathbf{a}^{*}+i C_{\rho}$, one of the functions $V_{i_{1}}, \ldots, V_{i_{k}}$ must be $V_{0}$. Thus after reordering if necessary, we may assume $V_{i_{1}}=V_{0}$. In this case $a_{1}(\lambda)$ is just the spherical Fourier transform of $f$.

If $f$ is a $K$-bi-invariant function, then $\pi_{\lambda}(f)$ is essentially the spherical Fourier transform (see the last line of (iii) above) and Theorem B (due to Ehrenpreis-Mautner and R. Krier) and its generalization to groups of arbitrary rank (Theorem 3.3 in [Si]) together tell us that the conjecture is verified in this case.

In the next part of this section we verify (a slightly weaker form of) the above conjecture for $G=\operatorname{SL}(2, R)$. For the rest of this section let $G=\mathrm{SL}(2, R)$ and $K=\mathrm{SO}(2)$. In this case $M=\{ \pm I\},\left\{V_{i}\right\}_{i=0}^{\infty}$ are just those characters of $K$ that agree on $\pm I$ and $V_{0}$ is the trivial character of $K$. In this case $a^{*}$ can be identified with $R$ via $Z \rightarrow Z \rho$ and with this identification $\mathbf{a}^{*}=R$ and $F=R+i[-1,1]$. Thus if $f \in L^{1}(X)$ is such that it is left $K$-finite and $\pi_{\lambda}(f)$ is nowhere vanishing on $F$, write

$$
\pi_{\lambda}(f) V_{0}=a_{1}(\lambda) V_{0}+a_{2}(\lambda) V_{i_{2}}+\cdots+a_{n}(\lambda) V_{i_{n}}
$$


(see remark (iii) following (C)), where $a_{1}, a_{2}, \ldots, a_{n}$ are non-trivial bounded holomorphic functions in $F^{0}$ and continuous on $F$. Then we have the following theorem:

THEOREM 4.1. Let $f, a_{1}, a_{2}, \ldots, a_{n}$ be as above. Assume that for some fixed (and small) $\varepsilon>0, a_{1}, a_{2}, \ldots, a_{n}$ extend to bounded holomorphic functions in $F_{\varepsilon}^{0}$. Further assume that for $\lambda \in F_{\varepsilon}^{0}$,

$$
\left|a_{1}(\lambda)\right|+\left|a_{2}(\lambda)\right|+\cdots+\left|a_{n}(\lambda)\right| \geq K\left|e^{-\lambda^{2 l}}\right|
$$

for some positive constant $K$ and positive integer $l$. Then the left $G$ translates of $f$ span a dense subspace of $L^{1}(X)$.

Proof. (We will use some facts about Harmonic Analysis on $\operatorname{SL}(2, R)$ from [EM1,2,3]. (However the reader is warned that the parametrization used by Ehrenpreis-Mautner is different from ours.) For a fixed character $V$ on $K$ let $L^{1}(V \backslash G / K)$ denote the subspace of functions $f$ in $L^{1}(G)$ such that $f\left(k_{1} x k_{2}\right)=V\left(k_{1}\right) f(x), \forall k_{1}, k_{2} \in K$. Similarly let $L^{1}(K \backslash G / V)$ be the collection of integrable functions $f$ such that

$$
f\left(k_{1} x k_{2}\right)=V\left(k_{2}\right) f(x), \quad \forall k_{1}, k_{2} \in K
$$

Now consider the orthonormal basis $\left\{V_{0}, V_{1}, V_{2}, \ldots\right\}$ of $L^{2}(K / M)$ and as explained before write

$$
\pi_{\lambda}(f) V_{0}=a_{1}(\lambda) V_{0}+a_{2}(\lambda) V_{i_{2}}+\cdots+a_{n}(\lambda) V_{i_{n}} .
$$

(See statement of Theorem 4.1 and the discussion immediately preceding the theorem.))

If $f \in L^{1}(K \backslash G / V)$, then its "Fourier transform" $a(\lambda)$ defined by $\pi_{\lambda}(f) V=a(\lambda) V_{0}$ will satisfy a certain transformation rule

$$
a(-\lambda)=(p(\lambda) / p(-\lambda)) a(\lambda)
$$

for a certain explicitly computable polynomial $p(\lambda)$ (which will of course depend on $V$ )-see [EM1,2,3] for details. Then using the work in [EM1,2,3] characterizing the Fourier transforms of rapidly decreasing functions, one can find functions $g_{k}$ in the Harish-ChandraSchwartz subspace of rapidly decreasing function of $L^{1}\left(K \backslash G / V_{i_{k}}\right)$ such that the Fourier transform of $g_{k}$ is given by $p_{k}(\lambda) e^{-\lambda^{2 l}}$. Here $p_{k}(\lambda)$ is a certain polynomial and (provided we choose $\varepsilon$ sufficiently small) 
$p_{k}(\lambda)$ has no zero in $F_{\varepsilon}$ except possibly at $+i$. Also $p_{1}(\lambda) \equiv 1$ (where $\left.V_{i_{1}}=V_{0}\right)$. Further $g_{k} * f$ is a $K$-bi-invariant function whose spherical Fourier transform is precisely $p_{k}(\lambda) e^{-\lambda^{2 l}} a_{k}(\lambda)$. Now consider the $n$ $K$-bi-invariant $L^{1}$-functions (i.e. functions in $I_{1}(G)$ ):

$$
g_{1} * f, \ldots, g_{n} * f
$$

whose spherical Fourier transforms are given by:

$$
\begin{aligned}
& p_{1}(\lambda) e^{-\lambda^{2 l}} a_{1}(\lambda) \quad\left(=e^{-\lambda^{2 l}} a_{1}(\lambda)\right), \\
& p_{2}(\lambda) e^{-\lambda^{2 l}} a_{2}(\lambda), \ldots, p_{n}(\lambda) e^{-\lambda^{2 l}} a_{n}(\lambda) .
\end{aligned}
$$

Also, as observed earlier $a_{2}(-i)=a_{3}(-i)=\cdots=a_{n}(-i)=0$. Hence $a_{1}(-i) \neq 0$. But since $a_{1}$ is an even function (being the spherical Fourier transform of $f), a_{1}(i)=a_{1}(-i) \neq 0$. Also $p_{1}(\lambda) \equiv 1$ and so $p_{1}(-i)=p_{1}(i) \neq 0$. The only possible zeros of $p_{k}$ in $F_{\varepsilon}$ are at $+i$ and also we have the estimate $\sum_{k=1}^{n}\left|a_{k}(\lambda)\right| \geq K\left|e^{-\lambda^{2 l}}\right|$. Putting all the above together we obtain that the new set of functions:

$$
p_{1}(\lambda) e^{-\lambda^{2 l}} a_{1}(\lambda), \ldots, p_{n}(\lambda) e^{-\lambda^{2 l}} a_{n}(\lambda)
$$

also satisfy a similar estimate in $F_{\varepsilon}^{0}$ i.e.

$$
\sum_{k=1}^{n}\left|p_{k}(\lambda) e^{-\lambda^{2 l}} a_{k}(\lambda)\right| \geq K^{\prime}\left|e^{-\lambda^{2 l^{\prime}}}\right|
$$

and so by Theorem 3.1 , it follows that the ideal generated by $g_{1} *$ $f, \ldots, g_{n} * f$ in $I_{1}(G)$ is dense in $I_{1}(G)$. However all the above functions are left convolutions against the single function $f$ and so it will follow easily that the left $G$-translates of $f$ span a dense subspace of $L^{1}(G / K)$-see the discussion in the beginning of this section. This completes the proof of Theorem 4.1.

The above proof relies on reducing the problem to the $K$-bi-invariant case and then using Theorem 3.1. This was achieved easily in this case because for $\operatorname{SL}(2, R)$, the $K$-types are all one dimensional. However we feel that with some (perhaps non-trivial) modification this method will also work for general rank-1 groups.

5. Concluding remarks. The problem dealt with in this paper is a kind of spectral synthesis problem for $L^{1}(G / K)$. As is evident from this paper, this kind of problem seems quite difficult-this is not altogether surprising considering the difficulty even in $L^{1}(R)$. For a detailed treatment of spectral synthesis for $I_{1}(G)$ see [K2]. 
However the space $C^{\infty}(G / K)$ seems much more tractable (at least in the case of rank-1). See [BaS], [BeS], [BeZ], [Waw] etc. The papers of Berenstein-Zalcman [BeZ] and Berenstein-Shahshahani [BeS] are particularly noteworthy and give interesting connections between the Pompeiu Problem and spectral synthesis in $C^{\infty}(G / K)$.

If one considers compact symmetric spaces, then the analogue of Wiener's theorem does hold. More precisely, let $G / K$ be a compact symmetric space (for a suitable compact connected group $G$ and closed subgroup $K \subset G$ ). Then we claim the following theorem is implicit in the work of Berenstein-Zalcman [BeZ] (though not quite stated in this fashion):

Proposition 5.1. Let $f \in L^{1}(G / K)$. If for each class-1 irreducible representation $\pi$ of $G, \pi(f) \neq 0$ (as an operator), then the left $G$ translates of $f$ span a dense subspace of $L^{1}(G / K)$.

(Of course a direct proof of the above can be given using properties of $L^{1}(K \backslash G / K)$ and the Peter-Weyl theorem. A representation $(\pi, H)$ of $G$ is said to be class-1 if $\exists V \neq 0$ in $H$ such that $\pi(k) V=V$, $\forall k \in K$.)

Now a few words about non-compact symmetric spaces of the Euclidean type i.e. $R^{n}$ with the usual Euclidean structure. For simplicity let us take $n=2$ i.e. $R^{2}$. Then $R^{2}$ can be viewed as $M(2) / \operatorname{SO}(2)$ where $M(2)$ is the group of (proper) rigid motions of the plane and $\mathrm{SO}(2)$ the group of rotations. Let $\left\{\pi_{\lambda}\right\}_{\lambda \in R^{+}}$be the principal series of representations of $M(2)$-see [Su] for details. Then:

Proposition 5.2. If $f \in L^{1}\left(M(2) / \mathrm{SO}(2)\right.$ ) and $\pi_{\lambda}(f) \neq 0$ (as an operator) for each $\lambda \in R^{+}$and $1(f) \neq 0$ (where 1 denotes the trivial one dimensional representation of $M(2)$ ), then the left $G$-translates of $f$ span a dense subspace of $L^{1}(M(2) / \mathrm{SO}(2)$ ) (i.e. the rigid motion translates of $f$ span a dense subspace of $L^{1}\left(R^{2}\right)$-if we view $M(2) / \mathrm{SO}(2)$ as $R^{2}$ and $f$ as an $L^{1}$-function on $R^{2}$ ).

This follows from the fact that if one views $f$ as a function on $R^{2}$, then the given condition is equivalent to: $\hat{f}(0) \neq 0$ and for each $\lambda \in R^{+}, \exists x_{\lambda}$ in $R^{2}$ with $\left\|x_{\lambda}\right\|=\lambda$ and $\hat{f}\left(x_{\lambda}\right) \neq 0$. (Here ${ }^{\wedge}$ denotes the usual Euclidean Fourier transform.) Our claim then follows from a slight variation of the usual Wiener-Tauberian theorem-for instance 
one can use Theorem 9.3 in [R]. Alternately, this will follow from a more general fact proved in Proposition 6.1 in [BST].

A (two sided) analogue of Wiener's theorem (due to R. Gangolli ([Gan]) is known for the full motion group. (See also [We].) So it would be interesting to have a modified version of Wiener's theorem for the full semi-simple Lie group $G$.

Finally a few words about the corresponding problem for $L^{2}$-functions on symmetric spaces of the non-compact type. As observed in [EM1] and [Si], if $f \in L^{2}(K \backslash G / K)$ and the spherical Fourier transform $\hat{f}$ of $f$ is non-zero a.e. $(\mu)$ on $\mathbf{a}^{*}$ (where $\mu$ is the HarishChandra Plancherel measure on $\left.\mathbf{a}^{*}\right)$, then $\left\{g * f: g \in C_{c}^{\infty}(K \backslash G / K)\right\}$ is dense in $L^{2}(K \backslash G / K)$. This easily leads to the following: If $f \in$ $L^{1}(G / K) \cap L^{2}(G / K)$ and $\pi_{\lambda}(f) \neq 0$ except possibly for $\lambda$ in a set of zero $\mu$-measure on $\mathbf{a}^{*}$, then $\left.\operatorname{Sp}^{g} f: g \in G\right\}$ is dense in $L^{2}(G / K)$. However because of the holomorphy of the spherical Fourier transform of $L^{1}$-functions in a certain tube in $\mathbf{a}_{c}^{*}$ around $\mathbf{a}^{*}$, one can easily prove that if $f$ is a non-trivial function in $L^{1}(G / K)$, the set $\left\{\lambda: \pi_{\lambda}(f)=0\right\}$ has zero $\mu$-measure. This combined with the observations made above leads to:

Proposition 5.3. Let $X(=G / K)$ be a symmetric space of the noncompact type and $f$ a non trivial function in $L^{1}(G / K) \cap L^{2}(G / K)$. Then $\operatorname{Sp}\left\{{ }^{g} f: g \in G\right\}$ is dense in $L^{2}(G / K)$.

In view of this a natural question to ask is: Suppose $G$ is a locally compact unimodular group, $\hat{G}$ the unitary dual of $G$ and $\mu$ the Plancherel measure on $\hat{G}$. If $f \in L^{1}(G) \cap L^{2}(G)$ has the property that for almost all $\pi$ (with respect to $\mu$ ) in $\hat{G}, \pi(f) \neq 0$, then is the smallest closed linear subspace containing all the two-sided translates of $f$ equal to $L^{2}(G)$ ? This question (in fact a more general version of this) has been settled in the affirmative by C. E. Sutherland [Sut]. Also in his paper the $L^{2}$-version of Wiener's theorem for general Gelfand pairs $(G, K)$ is discussed.

Acknowledgments. Don Marshall pointed out to the author the connection between the Corona theorem and the problem considered in this paper. This connection is crucial to our paper and the author is very grateful to Don Marshall for telling him about it. The author thanks Ramesh Gangolli for useful conversations and Somesh Bagchi for lecturing to him on the Corona Problem. 


\section{REFERENCES}

[BST] L. Brown, B. M. Schreiber and B. A. Taylor, Spectral synthesis and the Pompeiu problem, Ann. Inst. Fourier (Grenoble), 23 (1973), 125-154.

[BaS] S. C. Bagchi and A. Sitaram, Spherical mean periodic functions on semi-simple Lie groups, Pacific J. Math., 84 (1979), 241-250.

[BeS] C. A. Berenstein and M. Shahshahani, Harmonic analysis and the Pompeiu problem, Amer. J. Math., 105 (1983), 1217-1229.

[BeZ] C. A. Berenstein and L. Zalcman, Pompeiu's problem on symmetric spaces, Comment. Math. Helvetici, 55 (1980), 593-621.

[C] M. Cowling, Unitary and uniformly bounded representations of some simple Lie groups, in Harmonic Analysis and Group Representations, C.I.M.E (1980), 49-128.

[EM1] L. Ehrenpreis and F. I. Mautner, Some properties of the Fourier transform on semi-simple Lie groups-I, Ann. of Math., 61 (1955), 406-439.

[EM2] _ Some properties of the Fourier transform on semi-simple Lie groups-II, Trans. Amer. Math. Soc., 84 (1957), 1-55.

[EM3] _ Some properties of the Fourier transform on semi-simple Lie groupsIII, Trans. Amer. Math. Soc., 901 (1959), 431-484.

[Gan] R. Gangolli, On the symmetry of $L_{1}$ algebras of locally compact motion groups and the Wiener Tauberian theorem, J. Funct. Anal., 25 (1977), 244-252.

[Gar] J. B. Garnett, Bounded Analytic Functions, Academic Press, 1981.

[H1] S. Helgason, Differential Geometry and Symmetric Spaces, Academic Press, 1962.

[H2] _ Groups and Geometric Analysis, Academic Press, 1984.

[K1] R. Krier, Ph.D. thesis, University of Nancy, 1973.

[K2] _ Sur les idéaux de l'algèbre de Banach des fonctions integrables biinvariantes, Osaka J. Math., 12 (1975), 745-765.

[R] W. Rudin, Functional Analysis, McGraw-Hill, 1973.

[Si] A. Sitaram, An analogue of the Wiener-Tauberian theorem for spherical transforms on semi-simple Lie groups, Pacific J. Math., 89 (1980), 439-445.

[St] R. J. Stanke, Analytic uniformly bounded representations of $\mathrm{SU}(1, n+1)$, Trans. Amer. Math. Soc., 290 (1985), 281-302.

[Su] M. Sugiura, Unitary Representations and Harmonic Analysis, Kodansha, 1975.

[Sut] C. E. Sutherland, $L^{2}$-versions of Wiener's Tauberian theorems, preprint.

[TV] P. Trombi and V. S. Varadarajan, Spherical transforms on semi-simple Lie groups, Ann. of Math., 94 (1971), 246-303.

[War] G. Warner, Harmonic Analysis on Semi Simple Lie Groups, Vols. I and II, Springer-Verlag, 1972.

[Waw] A. Wawrzyñczyk, Spectral analysis and synthesis on symmetric spaces, preprint.

[We] Y. Weit, On the one-sided Wiener's theorem for the motion group, Ann. of Math., 111 (1980), 415-422.

Received March 28, 1987.

Indian Statistical Institute-Bangalore Centre

8Th Mile, Mysore RoAd

R. V. College Post Office

BANGALORE-560 059, INDIA 


\section{PACIFIC JOURNAL OF MATHEMATICS}

\section{EDITORS}

V. S. VARADARAJAN

(Managing Editor)

University of California

Los Angeles, CA 90024

Herbert Clemens

University of Utah

Salt Lake City, UT 84112

R. FINN

Stanford University

Stanford, CA 94305
HERMANN FLASCHKA

University of Arizona

Tucson, AZ 85721

RAMESh A. GANGOLLI

University of Washington Seattle, WA 98195

VAUGHAN F. R. JONES

University of California

Berkeley, CA 94720
ROBION KIRBY

University of California

Berkeley, CA 94720

C. C. MOORE

University of California

Berkeley, CA 94720

HAROLD STARK

University of California, San Diego

La Jolla, CA 92093

\section{ASSOCIATE EDITORS}
R. AREnS
E. F. BECKENBACH
B. H. NEUMANN
F. WOLF
K. YOSHIDA (1906-1982)

\section{SUPPORTING INSTITUTIONS}

UNIVERSITY OF ARIZONA

UNIVERSITY OF OREGON

UNIVERSITY OF BRITISH COLUMBIA UNIVERSITY OF SOUTHERN CALIFORNIA

CALIFORNIA INSTITUTE OF TECHNOLOGY

UNIVERSITY OF CALIFORNIA

STANFORD UNIVERSITY

MONTANA STATE UNIVERSITY

UNIVERSITY OF HAWAII

UNIVERSITY OF NEVADA, RENO

UNIVERSITY OF TOKYO

NEW MEXICO STATE UNIVERSITY

UNIVERSITY OF UTAH

OREGON STATE UNIVERSITY

WASHINGTON STATE UNIVERSITY

UNIVERSITY OF WASHINGTON 


\section{Pacific Journal of Mathematics}

\section{Vol. 133, No. $1 \quad$ March, 1988}

John Anderson, Finitely generated algebras and algebras of solutions to partial differential equations $\ldots \ldots \ldots \ldots \ldots \ldots \ldots \ldots \ldots \ldots \ldots \ldots \ldots$

Junichi Aramaki, On an extension of the Ikehara Tauberian theorem . . . . . 13

Giacomo Monti Bragadin, Abstract Riemannian stratifications .......... 31

Lawrence James Brenton and Richard Hill, On the Diophantine equation

$1=\sum 1 / n_{i}+1 / \prod n_{i}$ and a class of homologically trivial complex

surface singularities .................................41

C. Bruce Hughes, Controlled homotopy topological structures $\ldots . \ldots \ldots \ldots 69$

Peter Wilcox Jones and Takafumi Murai, Positive analytic capacity but zero Buffon needle probability . ...........................999

Gary M. Lieberman, Hölder continuity of the gradient at a corner for the

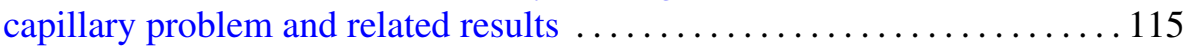

Feng Luo, Representing homology classes of $C \mathbf{P}^{2} \overline{C \mathbf{P}^{2}}$

Claudio Nebbia, Groups of isometries of a tree and the Kunze-Stein

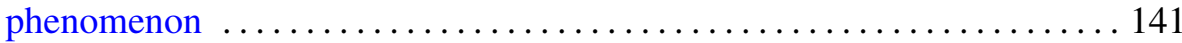

Stefan Richter, Unitary equivalence of invariant subspaces of Bergman and

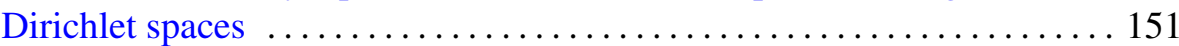

Paul Frederick Ringseth, The Selberg trace formula for groups without Eisenstein series ..................................... 157

Abderrazzak Sersouri, The Mazur property for compact sets $\ldots \ldots \ldots \ldots 185$ Alladi Sitaram, On an analogue of the Wiener Tauberian theorem for symmetric spaces of the noncompact type 